\title{
Representations by quaternary quadratic forms whose coefficients are 1, 3 and 9
}

by

\author{
Ayşe Alaca (Ottawa)
}

1. Introduction. For $n \in \mathbb{N}$ we set

$$
\sigma(n)=\sum_{d \mid n} d,
$$

where $d$ runs through the positive divisors of $n$. If $n \notin \mathbb{N}$ we set $\sigma(n)=0$.

For $a, b, c, d \in \mathbb{N}$ and $n \in \mathbb{N} \cup\{0\}$, we define

$$
N(a, b, c, d ; n)=\operatorname{card}\left\{(x, y, z, t) \in \mathbb{Z}^{4} \mid n=a x^{2}+b y^{2}+c z^{2}+d t^{2}\right\} .
$$

Clearly

$$
N(a, b, c, d ; 0)=1 .
$$

For $q \in \mathbb{C}$ with $|q|<1$ we have

$$
\sum_{n=0}^{\infty} N(a, b, c, d ; n) q^{n}=\varphi\left(q^{a}\right) \varphi\left(q^{b}\right) \varphi\left(q^{c}\right) \varphi\left(q^{d}\right),
$$

where $\varphi(q)$ denotes Ramanujan's theta function,

$$
\varphi(q):=\sum_{n=-\infty}^{\infty} q^{n^{2}}
$$

There are ten quaternary quadratic forms $a x^{2}+b y^{2}+c z^{2}+d t^{2}$ with $a, b, c, d \in$ $\{1,3,9\}$ and $1=a \leq b \leq c \leq d$. Formulae for $N(a, b, c, d ; n)(n \in \mathbb{N})$ with $(a, b, c, d)=(1,1,1,1),(1,1,1,3),(1,1,3,3),(1,3,3,3)$ appear in the literature (see [1], [2]). In this paper we treat the remaining six forms $(1,1,1,9)$, $(1,1,3,9),(1,1,9,9),(1,3,3,9),(1,3,9,9)$ and $(1,9,9,9)$.

2000 Mathematics Subject Classification: Primary 11E25.

Key words and phrases: sum of divisors function, quaternary quadratic forms, representations, theta functions, $(p, k)$-parametrization. 
Definition 1.1. For $k \in \mathbb{N}$ and $q \in \mathbb{C}$ with $|q|<1$, we define

$$
E_{k}=E_{k}(q):=\prod_{n=1}^{\infty}\left(1-q^{k n}\right) .
$$

The infinite product representation of $\varphi(q)$ is due to Jacobi [7]:

$$
\varphi(q)=\frac{E_{2}^{5}}{E_{1}^{2} E_{4}^{2}} .
$$

Definition 1.2. For $n \in \mathbb{N}$ we set

(a)

$$
\begin{aligned}
& A(n):=\sum_{d \mid n} d\left(\frac{12}{n / d}\right)=\sum_{d \mid n} \frac{n}{d}\left(\frac{12}{d}\right), \\
& B(n):=\sum_{d \mid n} d\left(\frac{-3}{d}\right)\left(\frac{-4}{n / d}\right)=\sum_{d \mid n} \frac{n}{d}\left(\frac{-3}{n / d}\right)\left(\frac{-4}{d}\right), \\
& C(n):=\sum_{d \mid n} d\left(\frac{-3}{n / d}\right)\left(\frac{-4}{d}\right)=\sum_{d \mid n} \frac{n}{d}\left(\frac{-3}{d}\right)\left(\frac{-4}{n / d}\right), \\
& D(n):=\sum_{d \mid n} d\left(\frac{12}{d}\right)=\sum_{d \mid n} \frac{n}{d}\left(\frac{12}{n / d}\right) .
\end{aligned}
$$

It is shown in $[2$, Sections 4 and 8$]$ for $n \in \mathbb{N}$ that

$$
\begin{aligned}
& N(1,1,1,3 ; n)=6 A(n)-2 B(n)+3 C(n)-D(n), \\
& N(1,3,3,3 ; n)=2 A(n)+2 B(n)-C(n)-D(n) .
\end{aligned}
$$

We determine an explicit formula for $N(1,3,3,9 ; n)$ in terms of $\sigma(n / k)(k \in$ $\{1,2,3,4,6,12\})$ by using the formula for $N(1,1,3,3 ; n)$ proved in $[1, \mathrm{p} .297]$ (see Theorem 1.1). We use the formulae for $N(1,1,1,3 ; n)$ and $N(1,3,3,3 ; n)$ proved in [2, pp. 228, 231] to determine explicit formulae for $N(1,1,3,9 ; n)$ and $N(1,3,9,9 ; n)$ in terms of $A(n), B(n), C(n)$ and $D(n)$ (see Theorems 1.2 and 1.3). Finally, we use the $(p, k)$-parametrization of $\varphi(q)$ given in [3] and [5] to determine explicit formulae for $N(1,1,1,9 ; n), N(1,1,9,9 ; n)$ and $N(1,9,9,9 ; n)$ in terms of $\sigma(n / k)(k \in\{1,2,3,6,9,36\})$ and the integers $c(n)(n \in \mathbb{N})$ defined by

$$
\sum_{n=1}^{\infty} c(n) q^{n}=q E_{6}^{4}
$$

(see Theorems $1.4,1.5$ and 1.6$)$. Clearly $c(n)=0$ if $n \not \equiv 1(\bmod 6)$. It follows from [8, Vol. II, p. 374] (see also [11, p. 121]) that 


$$
c(n)=\frac{1}{3} \sum_{\substack{x, y=-\infty \\ n=x^{2}+3 x y+3 y^{2} \\ x \equiv 2(\bmod 3) \\ y}}^{\infty 1(\bmod 2)}
$$

A numerical study showed that $c(n)$ cannot be expressed linearly in terms of $\sigma(n), A(n), B(n), C(n)$ and $D(n)$ when $n \equiv 1(\bmod 6)$.

THEOREM 1.1. For $n \in \mathbb{N}$,

$$
N(1,3,3,9 ; n)= \begin{cases}4 \sigma(n)-8 \sigma(n / 2)-12 \sigma(n / 3) & \\ +16 \sigma(n / 4)+24 \sigma(n / 6)-48 \sigma(n / 12) & \text { if } n \equiv 0(\bmod 3), \\ 2 \sigma(n)-4 \sigma(n / 2)+8 \sigma(n / 4) & \text { if } n \equiv 1(\bmod 3), \\ 0 & \text { if } n \equiv 2(\bmod 3) .\end{cases}
$$

TheOrem 1.2. For $n \in \mathbb{N}$,

$$
\begin{aligned}
N & (1,3,9,9 ; n) \\
& = \begin{cases}2 A(n / 3)+2 B(n / 3)-C(n / 3)-D(n / 3) & \text { if } n \equiv 0(\bmod 3), \\
2 A(n)-\frac{2}{3} B(n)+C(n)-\frac{1}{3} D(n) & \text { if } n \equiv 1(\bmod 3), \\
0 & \text { if } n \equiv 2(\bmod 3) .\end{cases}
\end{aligned}
$$

TheOREM 1.3. For $n \in \mathbb{N}$,

$N(1,1,3,9 ; n)$

$$
= \begin{cases}2 A(n / 3)+2 B(n / 3)-C(n / 3)-D(n / 3) & \text { if } n \equiv 0(\bmod 3), \\ 4 A(n)-\frac{4}{3} B(n)+2 C(n)-\frac{2}{3} D(n) & \text { if } n \equiv 1(\bmod 3), \\ 2 A(n)-\frac{2}{3} B(n)+C(n)-\frac{1}{3} D(n) & \text { if } n \equiv 2(\bmod 3) .\end{cases}
$$

TheOREM 1.4. For $n \in \mathbb{N}$,

$$
N(1,1,1,9 ; n)= \begin{cases}2 \sigma(n)+4 c(n) & \text { if } n \equiv 1(\bmod 6), \\ 12 \sigma(n)-24 \sigma(n / 2) & \text { if } n \equiv 2(\bmod 6), \\ 8 \sigma(n / 3) & \text { if } n \equiv 3(\bmod 6), \\ 6 \sigma(n)-12 \sigma(n / 2) & \text { if } n \equiv 4(\bmod 6), \\ 4 \sigma(n) & \text { if } n \equiv 5(\bmod 6), \\ 24 \sigma(n / 3)-48 \sigma(n / 6) & \text { if } n \equiv 0(\bmod 6) .\end{cases}
$$


TheOREM 1.5. For $n \in \mathbb{N}$,

$$
N(1,1,9,9 ; n)= \begin{cases}\frac{4}{3} \sigma(n)+\frac{8}{3} c(n) & \text { if } n \equiv 1(\bmod 6), \\ 4 \sigma(n)-8 \sigma(n / 2) & \text { if } n \equiv 2,4(\bmod 6), \\ 8 \sigma(n / 9) & \text { if } n \equiv 3(\bmod 6), \\ \frac{4}{3} \sigma(n) & \text { if } n \equiv 5(\bmod 6), \\ 8 \sigma(n / 9)-32 \sigma(n / 36) & \text { if } n \equiv 0(\bmod 6) .\end{cases}
$$

TheOREM 1.6. For $n \in \mathbb{N}$,

$$
N(1,9,9,9 ; n)= \begin{cases}\frac{2}{3} \sigma(n)+\frac{4}{3} c(n) & \text { if } n \equiv 1(\bmod 6), \\ 0 & \text { if } n \equiv 2,5(\bmod 6), \\ 8 \sigma(n / 9) & \text { if } n \equiv 3(\bmod 6), \\ 2 \sigma(n)-4 \sigma(n / 2) & \text { if } n \equiv 4(\bmod 6), \\ 8 \sigma(n / 9)-32 \sigma(n / 36) & \text { if } n \equiv 0(\bmod 6) .\end{cases}
$$

Theorems 1.1-1.3 appear to be new. In [12] Petr considered representations by the forms $x^{2}+y^{2}+z^{2}+9 t^{2}, x^{2}+y^{2}+9 z^{2}+9 t^{2}, x^{2}+9 y^{2}+9 z^{2}+9 t^{2}$. A different formulation of Theorem 1.4 has been given by Lomadze [10, Theorem 1a, p. 120]. Theorem 1.5 is due to Lomadze [9, p. 161], who obtained it using the theory of modular functions. Our approach is more elementary.

We close this introduction by recalling the $(p, k)$-parametrization of theta functions introduced in [3]. We then use it to obtain the $(p, k)$-parametrization of $\sum_{n=1}^{\infty} c(n) q^{n}$. As in [3, p. 178] we set

$$
\begin{aligned}
& p(q):=\frac{\varphi^{2}(q)-\varphi^{2}\left(q^{3}\right)}{2 \varphi^{2}\left(q^{3}\right)}, \\
& k(q):=\frac{\varphi^{3}\left(q^{3}\right)}{\varphi(q)} .
\end{aligned}
$$

When there is no risk of confusion we write $p$ for $p(q)$ and $k$ for $k(q)$. The following results were proved in $[3$, Theorems 9,10$]$.

Duplication Principle.

$$
\begin{aligned}
& p\left(q^{2}\right)=\frac{1+p-p^{2}-((1-p)(1+p)(1+2 p))^{1 / 2}}{p^{2}} \\
& k\left(q^{2}\right)=\frac{\left(1+p-p^{2}+((1-p)(1+p)(1+2 p))^{1 / 2}\right) k}{2} .
\end{aligned}
$$


TRIPLICATION PRINCIPLE.

$$
\begin{aligned}
p\left(q^{3}\right)=3^{-1} & ( \\
+ & \left.-4-3 p+6 p^{2}+4 p^{3}\right) \\
& 2^{2 / 3}\left(1-2 p-2 p^{2}\right)((1-p)(1+2 p)(2+p))^{1 / 3} \\
& \left.+2^{1 / 3}(1+2 p)((1-p)(1+2 p)(2+p))^{2 / 3}\right), \\
k\left(q^{3}\right)=3^{-2} & \left(3+2^{2 / 3}(1+2 p)((1-p)(1+2 p)(2+p))^{1 / 3}\right. \\
& \left.+2^{4 / 3}((1-p)(1+2 p)(2+p))^{2 / 3}\right) k .
\end{aligned}
$$

Ramanujan's discriminant function $\Delta(q)$ is defined by

$$
\Delta(q):=q E_{1}^{24}
$$

(see $[13$, eq. (92)], [14, p. 151]). It was shown in [4, eq. (3.32)] that

$$
\Delta\left(q^{6}\right)=\frac{1}{256} p^{6}(1+p)^{6}(1-p)^{2}(1+2 p)^{2}(2+p)^{2} k^{12} .
$$

By (1.9), (1.12) and (1.13), we obtain

$$
\begin{aligned}
\sum_{n=1}^{\infty} c(n) q^{n} & =q E_{6}^{4}=\left(q^{6} E_{6}^{24}\right)^{1 / 6}=\Delta\left(q^{6}\right)^{1 / 6} \\
& =2^{-4 / 3} p(1+p)(1-p)^{1 / 3}(1+2 p)^{1 / 3}(2+p)^{1 / 3} k^{2} .
\end{aligned}
$$

Using $p(1+p)=2^{-2}\left((1+2 p)^{2}-1\right)$, we obtain the following result.

LEMMA 1.1.

$$
\begin{aligned}
\sum_{n=1}^{\infty} c(n) q^{n}= & 2^{-10 / 3}(1-p)^{1 / 3}(1+2 p)^{7 / 3}(2+p)^{1 / 3} k^{2} \\
& -2^{-10 / 3}(1-p)^{1 / 3}(1+2 p)^{1 / 3}(2+p)^{1 / 3} k^{2} .
\end{aligned}
$$

2. Identities involving $\varphi$. In this section we obtain the $(p, k)$-parametrization of $\varphi\left(q^{9}\right)$ (Theorem 2.2) and deduce some identities involving $\varphi(q), \varphi\left(q^{3}\right)$ and $\varphi\left(q^{9}\right)$, which are used in Sections 6-8 (see Theorems 2.3$2.5)$.

It was shown in $[2$, eq. (2.3)] that

$$
\begin{aligned}
\varphi(q) & =(1+2 p)^{3 / 4} k^{1 / 2}, \\
\varphi\left(q^{3}\right) & =(1+2 p)^{1 / 4} k^{1 / 2} .
\end{aligned}
$$

It is shown in $[1$, p. 297] that

$$
\begin{aligned}
N(1,1,3,3 ; n)= & 4 \sigma(n)-8 \sigma(n / 2)-12 \sigma(n / 3)+16 \sigma(n / 4) \\
& +24 \sigma(n / 6)-48 \sigma(n / 12) .
\end{aligned}
$$

Our first theorem gives an alternative formulation of the second part of the triplication principle. 
THEOREM 2.1 .

$$
k\left(q^{3}\right)=\left(\frac{(1+2 p)^{2 / 3}+2^{2 / 3}(1-p)^{1 / 3}(2+p)^{1 / 3}}{3}\right)^{3} k .
$$

Proof. We have

$$
\begin{aligned}
\left(\frac{(1+}{2 p)^{2 / 3}+2^{2 / 3}(1-p)^{1 / 3}(2+p)^{1 / 3}}\right)^{3} k & \\
= & \frac{1}{27}\left((1+2 p)^{2}+3 \cdot 2^{2 / 3}(1-p)^{1 / 3}(1+2 p)^{4 / 3}(2+p)^{1 / 3}\right. \\
& \left.\quad+3 \cdot 2^{4 / 3}(1-p)^{2 / 3}(1+2 p)^{2 / 3}(2+p)^{2 / 3}+2^{2}(1-p)(2+p)\right) k \\
= & \frac{1}{27}\left(9+3 \cdot 2^{2 / 3}(1+2 p)((1-p)(1+2 p)(2+p))^{1 / 3}\right. \\
& \left.\quad+3 \cdot 2^{4 / 3}((1-p)(1+2 p)(2+p))^{2 / 3}\right) k \\
= & \frac{1}{9}\left(3+2^{2 / 3}(1+2 p)((1-p)(1+2 p)(2+p))^{1 / 3}\right. \\
& \left.\quad+2^{4 / 3}((1-p)(1+2 p)(2+p))^{2 / 3}\right) k \\
= & k\left(q^{3}\right)
\end{aligned}
$$

by the second part of the triplication principle.

Theorem 2.1 enables us to give the $(p, k)$-parametrization of $\varphi\left(q^{9}\right)$.

THEOREM 2.2 .

$$
\varphi\left(q^{9}\right)=\frac{1}{3}(1+2 p)^{1 / 12}\left((1+2 p)^{2 / 3}+2^{2 / 3}(1-p)^{1 / 3}(2+p)^{1 / 3}\right) k^{1 / 2} .
$$

Proof. Replacing $q$ by $q^{3}$ in (1.11) we obtain $\varphi^{3}\left(q^{9}\right)=\varphi\left(q^{3}\right) k\left(q^{3}\right)$. Then, by Theorem 2.1 and (2.2), we obtain

$$
\varphi^{3}\left(q^{9}\right)=(1+2 p)^{1 / 4} k^{1 / 2}\left(\frac{(1+2 p)^{2 / 3}+2^{2 / 3}(1-p)^{1 / 3}(2+p)^{1 / 3}}{3}\right)^{3} k .
$$

Taking the cube root of each side, we obtain the asserted formula.

THEOREM 2.3 .

$$
\varphi(q) \varphi^{3}\left(q^{9}\right)=\varphi^{2}(q) \varphi^{2}\left(q^{9}\right)-\frac{1}{3} \varphi^{3}(q) \varphi\left(q^{9}\right)+\frac{1}{3} \varphi^{4}\left(q^{3}\right) .
$$

Proof. This identity follows from (2.1), (2.2) and Theorem 2.2.

From Theorem 2.3 we obtain

$$
3 \frac{\varphi\left(q^{9}\right)}{\varphi(q)}=1+\left(\frac{9 \varphi^{4}\left(q^{3}\right)}{\varphi^{4}(q)}-1\right)^{1 / 3}
$$

which was proved in [6, p. 345].

Theorem 2.4 .

$$
\varphi^{2}(q) \varphi^{2}\left(q^{9}\right)=\frac{1}{6} \varphi^{4}(q)-\frac{2}{3} \varphi^{4}\left(q^{3}\right)+\frac{3}{2} \varphi^{4}\left(q^{9}\right)+\frac{8}{3} \sum_{n=1}^{\infty} c(n) q^{n} .
$$


Proof. By (2.1) and Theorem 2.2, we obtain

$$
\begin{aligned}
\varphi^{2}(q) \varphi^{2}\left(q^{9}\right)= & \frac{1}{9}(1+2 p)^{5 / 3}\left((1+2 p)^{4 / 3}+2^{5 / 3}(1-p)^{1 / 3}(1+2 p)^{2 / 3}(2+p)^{1 / 3}\right. \\
& \left.+2^{4 / 3}(1-p)^{2 / 3}(2+p)^{2 / 3}\right) k^{2} \\
= & \frac{1}{9}(1+2 p)^{3} k^{2}+\frac{1}{9} \cdot 2^{5 / 3}(1-p)^{1 / 3}(1+2 p)^{7 / 3}(2+p)^{1 / 3} k^{2} \\
& +\frac{1}{9} \cdot 2^{4 / 3}(1-p)^{2 / 3}(1+2 p)^{5 / 3}(2+p)^{2 / 3} k^{2} .
\end{aligned}
$$

On the other hand, by Lemma 1.1, (2.1), (2.2), and Theorem 2.2, we obtain

$$
\begin{aligned}
\frac{1}{6} \varphi^{4}(q)- & \frac{2}{3} \varphi^{4}\left(q^{3}\right)+\frac{3}{2} \varphi^{4}\left(q^{9}\right)+\frac{8}{3} \sum_{n=1}^{\infty} c(n) q^{n} \\
= & \frac{1}{6}(1+2 p)^{3} k^{2}-\frac{2}{3}(1+2 p) k^{2} \\
& +\frac{3}{2}\left(\frac{1}{27}\left(-8 p^{3}-12 p^{2}+18 p+11\right) k^{2}\right. \\
& \quad+\frac{1}{81} \cdot 2^{8 / 3}(1-p)^{1 / 3}(1+2 p)^{7 / 3}(2+p)^{1 / 3} k^{2} \\
& \quad+\frac{1}{27} \cdot 2^{7 / 3}(1-p)^{2 / 3}(1+2 p)^{5 / 3}(2+p)^{2 / 3} k^{2} \\
& \left.\quad+\frac{1}{81} \cdot 2^{8 / 3}(1-p)^{4 / 3}(1+2 p)^{1 / 3}(2+p)^{4 / 3} k^{2}\right) \\
& +\frac{8}{3}\left(2^{-10 / 3}(1-p)^{1 / 3}(1+2 p)^{7 / 3}(2+p)^{1 / 3} k^{2}\right. \\
& \left.\quad-2^{-10 / 3}(1-p)^{1 / 3}(1+2 p)^{1 / 3}(2+p)^{1 / 3} k^{2}\right) \\
= & \frac{1}{9}\left(1+6 p+12 p^{2}+8 p^{3}\right) k^{2} \\
& +\frac{1}{3}(1-p)^{1 / 3}(1+2 p)^{7 / 3}(2+p)^{1 / 3} k^{2} \\
& \times\left(\frac{2^{5 / 3}}{9}+\frac{2^{5 / 3}(1-p)(2+p)}{9(1+2 p)^{2}}+\frac{1}{2^{1 / 3}}-\frac{1}{2^{1 / 3}(1+2 p)^{2}}\right) \\
& +\frac{1}{9} \cdot 2^{4 / 3}(1-p)^{2 / 3}(1+2 p)^{5 / 3}(2+p)^{2 / 3} k^{2} \\
= & \frac{1}{9}(1+2 p)^{3} k^{2}+\frac{1}{9} \cdot 2^{5 / 3}(1-p)^{1 / 3}(1+2 p)^{7 / 3}(2+p)^{1 / 3} k^{2} \\
& +\frac{1}{9} \cdot 2^{4 / 3}(1-p)^{2 / 3}(1+2 p)^{5 / 3}(2+p)^{2 / 3} k^{2} .
\end{aligned}
$$

This completes the proof of Theorem 2.4.

The following identity is a classical identity due to Jacobi [7]:

$$
\varphi^{4}(q)=1+\sum_{n=1}^{\infty}(8 \sigma(n)-32 \sigma(n / 4)) q^{n} .
$$

Replacing $q$ by $q^{3}$ and $q^{9}$ in (2.6), we obtain

$$
\begin{aligned}
& \varphi^{4}\left(q^{3}\right)=1+\sum_{n=1}^{\infty}(8 \sigma(n / 3)-32 \sigma(n / 12)) q^{n}, \\
& \varphi^{4}\left(q^{9}\right)=1+\sum_{n=1}^{\infty}(8 \sigma(n / 9)-32 \sigma(n / 36)) q^{n} .
\end{aligned}
$$


The Eisenstein series $L(q)$ is defined by

$$
L(q):=1-24 \sum_{n=1}^{\infty} \sigma(n) q^{n} .
$$

It was shown in $[4$, eq. $(3.11)$, p. 33$]$ that

$$
L\left(q^{6}\right)-2 L\left(q^{12}\right)=-\left(1+2 p-p^{3}-\frac{1}{2} p^{4}\right) k^{2} .
$$

For $k \in \mathbb{N}$ and $i \in\{0,1, \ldots, k-1\}$ we define

$$
L_{i, k}(q):=\sum_{\substack{n=1 \\ n \equiv i(\bmod k)}}^{\infty} \sigma(n) q^{n} .
$$

It was shown in $[3$, eq. (14.6), p. 189] that

$$
L_{1,2}\left(q^{3}\right)=\left(\frac{1}{8} p^{3}+\frac{1}{16} p^{4}\right) k^{2},
$$

and in $[3$, p. 190] that

$$
\begin{aligned}
L_{1,3}(q) & +2 L_{2,3}(q)=\frac{1}{9}\left(1+8 p+18 p^{2}+8 p^{3}+p^{4}\right) k^{2} \\
& -\frac{1}{9} \cdot 2^{-1 / 3}\left(1+3 p-3 p^{2}-p^{3}\right)((1-p)(1+2 p)(2+p))^{1 / 3} k^{2} .
\end{aligned}
$$

Applying the duplication principle twice to $L_{1,3}(q)+2 L_{2,3}(q)$, we obtain

$$
\begin{aligned}
L_{1,3}\left(q^{4}\right)+ & 2 L_{2,3}\left(q^{4}\right)=\frac{1}{36}\left(-2-2 p+p^{2}\right)^{2} k^{2} \\
& +\frac{1}{72} \cdot 2^{2 / 3}\left(-4-6 p+p^{3}\right)((1-p)(1+2 p)(2+p))^{1 / 3} k^{2} .
\end{aligned}
$$

THEOREM 2.5 .

$$
\begin{aligned}
\varphi^{3}(q) \varphi\left(q^{9}\right)= & 2 L_{1,3}(q)+4 L_{2,3}(q)-8 L_{1,3}\left(q^{4}\right)-16 L_{2,3}\left(q^{4}\right) \\
& -L\left(q^{6}\right)+2 L\left(q^{12}\right)+8 L_{1,2}\left(q^{3}\right)+4 \sum_{n=1}^{\infty} c(n) q^{n} .
\end{aligned}
$$

Proof. By (2.1) and Theorem 2.2, we have

$$
\varphi^{3}(q) \varphi\left(q^{9}\right)=\frac{1}{3}(1+2 p)^{3} k^{2}+\frac{1}{3} \cdot 2^{2 / 3}(1-p)^{1 / 3}(1+2 p)^{7 / 3}(2+p)^{1 / 3} k^{2} .
$$

On the other hand, by (2.10), (2.12), (2.13), (2.14), and Lemma 1.1,

$$
\begin{aligned}
& 2 L_{1,3}(q)+4 L_{2,3}(q)-8 L_{1,3}\left(q^{4}\right)-16 L_{2,3}\left(q^{4}\right) \\
& -L\left(q^{6}\right)+2 L\left(q^{12}\right)+8 L_{1,2}\left(q^{3}\right)+4 \sum_{n=1}^{\infty} c(n) q^{n} \\
& =2\left(\frac{1}{9}\left(1+8 p+18 p^{2}+8 p^{3}+p^{4}\right) k^{2}\right. \\
& \left.\quad-\frac{1}{9} \cdot 2^{-1 / 3}\left(1+3 p-3 p^{2}-p^{3}\right)((1-p)(1+2 p)(2+p))^{1 / 3} k^{2}\right)
\end{aligned}
$$




$$
\begin{aligned}
& -8\left(\frac{1}{36}\left(-2-2 p+p^{2}\right)^{2} k^{2}\right. \\
& \left.+\frac{1}{72} \cdot 2^{2 / 3}\left(-4-6 p+p^{3}\right)((1-p)(1+2 p)(2+p))^{1 / 3} k^{2}\right) \\
& +\left(1+2 p-p^{3}-\frac{1}{2} p^{4}\right) k^{2}+8\left(\frac{1}{8} p^{3}+\frac{1}{16} p^{4}\right) k^{2} \\
& +4\left(2^{-10 / 3}(1-p)^{1 / 3}(1+2 p)^{7 / 3}(2+p)^{1 / 3} k^{2}\right. \\
& \left.-2^{-10 / 3}(1-p)^{1 / 3}(1+2 p)^{1 / 3}(2+p)^{1 / 3} k^{2}\right) \\
& =\frac{2}{9}\left(1+8 p+18 p^{2}+8 p^{3}+p^{4}\right) k^{2} \\
& -\frac{1}{9} \cdot 2^{2 / 3}\left(1+3 p-3 p^{2}-p^{3}\right)((1-p)(1+2 p)(2+p))^{1 / 3} k^{2} \\
& -\frac{2}{9}\left(4+8 p-4 p^{3}+p^{4}\right) k^{2} \\
& -\frac{1}{9} \cdot 2^{2 / 3}\left(-4-6 p+p^{3}\right)((1-p)(1+2 p)(2+p))^{1 / 3} k^{2}+(1+2 p) k^{2} \\
& +2^{-4 / 3}(1+2 p)^{2}((1-p)(1+2 p)(2+p))^{1 / 3} k^{2} \\
& -2^{-4 / 3}((1-p)(1+2 p)(2+p))^{1 / 3} k^{2} \\
& =\frac{1}{3}(1+2 p)^{3} k^{2} \\
& +((1-p)(1+2 p)(2+p))^{1 / 3} k^{2}\left(\frac{2^{2 / 3}\left(1+p+p^{2}\right)}{3}+\frac{(1+2 p)^{2}}{2^{4 / 3}}-\frac{1}{2^{4 / 3}}\right) \\
& =\frac{1}{3}(1+2 p)^{3} k^{2} \\
& +((1-p)(1+2 p)(2+p))^{1 / 3} k^{2} \frac{4(1+2 p)^{2}}{3 \cdot 2^{4 / 3}} \\
& =\frac{1}{3}(1+2 p)^{3} k^{2}+\frac{1}{3} \cdot 2^{2 / 3}(1-p)^{1 / 3}(1+2 p)^{7 / 3}(2+p)^{1 / 3} k^{2} .
\end{aligned}
$$

This completes the proof of Theorem 2.5.

3. Evaluation of $N(1,3,3,9 ; n)$ : Proof of Theorem 1.1. First, we prove the following lemma.

Lemma 3.1. Let $n \in \mathbb{N}$. Then

$$
N(1,3,3,9 ; n)= \begin{cases}N(1,1,3,3 ; n / 3) & \text { if } n \equiv 0(\bmod 3), \\ \frac{1}{2} N(1,1,3,3 ; n) & \text { if } n \equiv 1(\bmod 3), \\ 0 & \text { if } n \equiv 2(\bmod 3) .\end{cases}
$$

Proof. Let $n \equiv 0(\bmod 3)$. If $n=x^{2}+3 y^{2}+3 z^{2}+9 t^{2}$, then $x \equiv 0$ $(\bmod 3)$. Hence

$$
\begin{aligned}
N(1,3,3,9 ; n) & =\operatorname{card}\left\{(x, y, z, t) \in \mathbb{Z}^{4} \mid n=x^{2}+3 y^{2}+3 z^{2}+9 t^{2}\right\} \\
& =\operatorname{card}\left\{(x, y, z, t) \in \mathbb{Z}^{4} \mid n=x^{2}+3 y^{2}+3 z^{2}+9 t^{2}, x \equiv 0(\bmod 3)\right\} \\
& =\operatorname{card}\left\{\left(x_{1}, y, z, t\right) \in \mathbb{Z}^{4} \mid n=9 x_{1}^{2}+3 y^{2}+3 z^{2}+9 t^{2}\right\} \\
& =\operatorname{card}\left\{\left(y, z, x_{1}, t\right) \in \mathbb{Z}^{4} \mid n=3 y^{2}+3 z^{2}+9 x_{1}^{2}+9 t^{2}\right\} \\
& =\operatorname{card}\left\{\left(y, z, x_{1}, t\right) \in \mathbb{Z}^{4} \mid n / 3=y^{2}+z^{2}+3 x_{1}^{2}+3 t^{2}\right\} \\
& =N(1,1,3,3 ; n / 3) .
\end{aligned}
$$


Let $n \equiv 1(\bmod 3)$. If $n=x^{2}+y^{2}+3 z^{2}+3 t^{2}$, then $x^{2}+y^{2} \equiv 1(\bmod 3)$, and so either $x \equiv 0, y \not \equiv 0$ or $x \not \equiv 0, y \equiv 0$ modulo 3 . Hence

$$
\begin{aligned}
N & (1,1,3,3 ; n) \\
= & \operatorname{card}\left\{(x, y, z, t) \in \mathbb{Z}^{4} \mid n=x^{2}+y^{2}+3 z^{2}+3 t^{2}\right\} \\
= & \operatorname{card}\left\{(x, y, z, t) \in \mathbb{Z}^{4} \mid n=x^{2}+y^{2}+3 z^{2}+3 t^{2}, x \equiv 0, y \not \equiv 0(\bmod 3)\right\} \\
& +\operatorname{card}\left\{(x, y, z, t) \in \mathbb{Z}^{4} \mid n=x^{2}+y^{2}+3 z^{2}+3 t^{2}, x \not \equiv 0, y \equiv 0(\bmod 3)\right\} \\
= & 2 \operatorname{card}\left\{\left(x_{1}, y, z, t\right) \in \mathbb{Z}^{4} \mid n=9 x_{1}^{2}+y^{2}+3 z^{2}+3 t^{2}, y \not \equiv 0(\bmod 3)\right\} \\
= & 2 \operatorname{card}\left\{\left(y, z, t, x_{1}\right) \in \mathbb{Z}^{4} \mid n=y^{2}+3 z^{2}+3 t^{2}+9 x_{1}^{2}\right\} \\
= & 2 N(1,3,3,9 ; n) .
\end{aligned}
$$

If $n \equiv 2(\bmod 3)$, then clearly $N(1,3,3,9 ; n)=0$, which completes the proof of Lemma 3.1.

Proof of Theorem 1.1. Let $n \equiv 0(\bmod 3)$. By Lemma 3.1 and $(2.3)$, we have

$$
\begin{aligned}
N(1,3,3,9 ; n)= & 4 \sigma(n / 3)-8 \sigma(n / 6)-12 \sigma(n / 9)+16 \sigma(n / 12) \\
& +24 \sigma(n / 18)-48 \sigma(n / 36) .
\end{aligned}
$$

On the other hand, for $n \equiv 0(\bmod 3)$ we have the elementary identities

$$
\begin{aligned}
& \sigma(n)-4 \sigma(n / 3)+3 \sigma(n / 9)=0, \\
& \sigma(n / 2)-4 \sigma(n / 6)+3 \sigma(n / 18)=0, \\
& \sigma(n / 4)-4 \sigma(n / 12)+3 \sigma(n / 36)=0 .
\end{aligned}
$$

By substituting (3.2)-(3.4) into (3.1), we obtain

$$
\begin{aligned}
N(1,3,3,9 ; n)= & 4 \sigma(n / 3)-8 \sigma(n / 6)-4(4 \sigma(n / 3)-\sigma(n))+16 \sigma(n / 12) \\
& +8(4 \sigma(n / 6)-\sigma(n / 2))-16(4 \sigma(n / 12)-\sigma(n / 4)) \\
= & 4 \sigma(n)-8 \sigma(n / 2)-12 \sigma(n / 3)+16 \sigma(n / 4) \\
& +24 \sigma(n / 6)-48 \sigma(n / 12),
\end{aligned}
$$

which completes the proof of Theorem 1.1 for $n \equiv 0(\bmod 3)$. When $n \equiv 1$ $(\bmod 3)$, the assertion follows from Lemma 3.1 and $(2.3)$. When $n \equiv 2$ $(\bmod 3)$, the assertion follows directly from Lemma 3.1.

4. Evaluation of $N(1,3,9,9 ; n)$ : Proof of Theorem 1.2. First, we prove the following lemma.

Lemma 4.1. Let $n \in \mathbb{N}$. Then

$$
N(1,3,9,9 ; n)= \begin{cases}N(1,3,3,3 ; n / 3) & \text { if } n \equiv 0(\bmod 3), \\ \frac{1}{3} N(1,1,1,3 ; n) & \text { if } n \equiv 1(\bmod 3), \\ 0 & \text { if } n \equiv 2(\bmod 3) .\end{cases}
$$


Proof. The case $n \equiv 2(\bmod 3)$ is trivial. The case $n \equiv 0(\bmod 3)$ can be treated in a manner similar to the proof of Lemma 3.1. Thus, we prove the lemma only when $n \equiv 1(\bmod 3)$. Clearly $n=x^{2}+y^{2}+z^{2}+3 t^{2} \equiv 1$ $(\bmod 3)$ if and only if $x^{2}+y^{2}+z^{2} \equiv 1(\bmod 3)$. The only possible cases are $x \equiv 0, y \equiv 0, z \not \equiv 0$ or $x \equiv 0, y \not \equiv 0, z \equiv 0$ or $x \not \equiv 0, y \equiv 0, z \equiv 0(\bmod 3)$. Hence

$$
\begin{aligned}
& N(1,1,1,3 ; n)=\operatorname{card}\left\{(x, y, z, t) \in \mathbb{Z}^{4} \mid n=x^{2}+y^{2}+z^{2}+3 t^{2}\right\} \\
& =\operatorname{card}\left\{(x, y, z, t) \in \mathbb{Z}^{4} \mid n=x^{2}+y^{2}+z^{2}+3 t^{2}, x \equiv 0, y \equiv 0, z \not \equiv 0(\bmod 3)\right\} \\
& \quad+\operatorname{card}\left\{(x, y, z, t) \in \mathbb{Z}^{4} \mid n=x^{2}+y^{2}+z^{2}+3 t^{2}, x \equiv 0, y \not \equiv 0, z \equiv 0(\bmod 3)\right\} \\
& \quad+\operatorname{card}\left\{(x, y, z, t) \in \mathbb{Z}^{4} \mid n=x^{2}+y^{2}+z^{2}+3 t^{2}, x \neq 0, y \equiv 0, z \equiv 0(\bmod 3)\right\} \\
& =3 \operatorname{card}\left\{\left(x_{1}, y_{1}, z, t\right) \in \mathbb{Z}^{4} \mid n=9 x_{1}^{2}+9 y_{1}^{2}+z^{2}+3 t^{2}, z \not \equiv 0(\bmod 3)\right\} \\
& =3 \operatorname{card}\left\{\left(z, t, x_{1}, y_{1}\right) \in \mathbb{Z}^{4} \mid n=z^{2}+3 t^{2}+9 x_{1}^{2}+9 y_{1}^{2}\right\} \\
& =3 N(1,3,9,9 ; n),
\end{aligned}
$$

so

$$
N(1,3,9,9 ; n)=\frac{1}{3} N(1,1,1,3 ; n), \quad n \equiv 1(\bmod 3) .
$$

The proof of Theorem 1.2 now follows from Lemma 4.1, (1.7) and (1.8).

5. Evaluation of $N(1,1,3,9 ; n)$ : Proof of Theorem 1.3. First, we prove the following lemma.

Lemma 5.1. Let $n \in \mathbb{N}$. Then

$$
N(1,1,3,9 ; n)= \begin{cases}N(1,3,3,3 ; n / 3) & \text { if } n \equiv 0(\bmod 3), \\ \frac{2}{3} N(1,1,1,3 ; n) & \text { if } n \equiv 1(\bmod 3), \\ \frac{1}{3} N(1,1,1,3 ; n) & \text { if } n \equiv 2(\bmod 3) .\end{cases}
$$

Proof. Let $n \equiv 0(\bmod 3)$. Then $n=x^{2}+y^{2}+3 z^{2}+9 t^{2} \equiv 0(\bmod 3)$ if and only if $x \equiv y \equiv 0(\bmod 3)$. Hence

$$
\begin{aligned}
& N(1,1,3,9 ; n)=\operatorname{card}\left\{(x, y, z, t) \in \mathbb{Z}^{4} \mid n=x^{2}+y^{2}+3 z^{2}+9 t^{2}\right\} \\
& \quad=\operatorname{card}\left\{(x, y, z, t) \in \mathbb{Z}^{4} \mid n=x^{2}+y^{2}+3 z^{2}+9 t^{2}, x \equiv y \equiv 0(\bmod 3)\right\} \\
& \quad=\operatorname{card}\left\{\left(x_{1}, y_{1}, z, t\right) \in \mathbb{Z}^{4} \mid n=9 x_{1}^{2}+9 y_{1}^{2}+3 z^{2}+9 t^{2}\right\} \\
& \quad=\operatorname{card}\left\{\left(z, x_{1}, y_{1}, t\right) \in \mathbb{Z}^{4} \mid n / 3=z^{2}+3 x_{1}^{2}+3 y_{1}^{2}+3 t^{2}\right\} \\
& \quad=N(1,3,3,3 ; n / 3) .
\end{aligned}
$$


Let $n \equiv 1(\bmod 3)$. Then $n=x^{2}+y^{2}+3 z^{2}+9 t^{2} \equiv 1(\bmod 3)$ if and only if $x^{2}+y^{2} \equiv 1(\bmod 3)$. Thus, either $x \equiv 0, y \not \equiv 0(\bmod 3)$, or $x \not \equiv 0$, $y \equiv 0(\bmod 3)$. Hence

$$
\begin{aligned}
& N \\
& \quad(1,1,3,9 ; n)=\operatorname{card}\left\{(x, y, z, t) \in \mathbb{Z}^{4} \mid n=x^{2}+y^{2}+3 z^{2}+9 t^{2}\right\} \\
& \quad=2 \operatorname{card}\left\{(x, y, z, t) \in \mathbb{Z}^{4} \mid n=x^{2}+y^{2}+3 z^{2}+9 t^{2}, x \equiv 0, y \not \equiv 0(\bmod 3)\right\} \\
& \quad=2 \operatorname{card}\left\{\left(x_{1}, y, z, t\right) \in \mathbb{Z}^{4} \mid n=9 x_{1}^{2}+y^{2}+3 z^{2}+9 t^{2}\right\} \\
& \quad=2 \operatorname{card}\left\{\left(y, z, x_{1}, t\right) \in \mathbb{Z}^{4} \mid n=y^{2}+3 z^{2}+9 x_{1}^{2}+9 t^{2}\right\} \\
& \quad=2 N(1,3,9,9 ; n) .
\end{aligned}
$$

We have

$$
N(1,1,3,9 ; n)=2 N(1,3,9,9 ; n), \quad n \equiv 1(\bmod 3) .
$$

Then, by (4.1) and (5.1), we obtain

$$
N(1,1,3,9 ; n)=\frac{2}{3} N(1,1,1,3 ; n), \quad n \equiv 1(\bmod 3) .
$$

Let $n \equiv 2(\bmod 3)$. Then $n=x^{2}+y^{2}+z^{2}+3 t^{2} \equiv 2(\bmod 3)$ if and only if $x \equiv 0, y \not \equiv 0, z \not \equiv 0$ or $x \not \equiv 0, y \equiv 0, z \not \equiv 0$ or $x \not \equiv 0, y \not \equiv 0, z \equiv 0(\bmod 3)$. Hence

$$
\begin{aligned}
& N(1,1,1,3 ; n)=\operatorname{card}\left\{(x, y, z, t) \in \mathbb{Z}^{4} \mid n=x^{2}+y^{2}+z^{2}+3 t^{2}\right\} \\
& =3 \operatorname{card}\left\{(x, y, z, t) \in \mathbb{Z}^{4} \mid n=x^{2}+y^{2}+z^{2}+3 t^{2},\right. \\
& \quad x \equiv 0, y \not \equiv 0, z \not \equiv 0(\bmod 3)\} \\
& =3 \operatorname{card}\left\{\left(x_{1}, y, z, t\right) \in \mathbb{Z}^{4} \mid n=9 x_{1}^{2}+y^{2}+z^{2}+3 t^{2}, y \not \equiv 0, z \not \equiv 0(\bmod 3)\right\} \\
& =3 \operatorname{card}\left\{\left(y, z, t, x_{1}\right) \in \mathbb{Z}^{4} \mid n=y^{2}+z^{2}+3 t^{2}+9 x_{1}^{2}\right\} \\
& =3 N(1,1,3,9 ; n),
\end{aligned}
$$

which completes the proof of Lemma 5.1.

Theorem 1.3 now follows from Lemma 5.1, (1.7) and (1.8).

6. Evaluation of $N(1,1,1,9 ; n)$ : Proof of Theorem 1.4. By Theorem 2.5 and $(1.4),(2.10)-(2.14)$ we obtain

$$
\begin{aligned}
\sum_{n=0}^{\infty} N(1,1,1,9 ; n) q^{n}= & 2 L_{1,3}(q)+4 L_{2,3}(q)-8 L_{1,3}\left(q^{4}\right)-16 L_{2,3}\left(q^{4}\right) \\
& -L\left(q^{6}\right)+2 L\left(q^{12}\right)+8 L_{1,2}\left(q^{3}\right)+4 \sum_{n=1}^{\infty} c(n) q^{n}
\end{aligned}
$$




$$
\begin{aligned}
& =2 \sum_{\substack{n=1 \\
n \equiv 1(\bmod 3)}}^{\infty} \sigma(n) q^{n}+4 \sum_{\substack{n=1 \\
n \equiv 2(\bmod 3)}}^{\infty} \sigma(n) q^{n}-8 \sum_{\substack{n=1 \\
n \equiv 1(\bmod 3)}}^{\infty} \sigma(n) q^{4 n} \\
& -16 \sum_{\substack{n=1 \\
n \equiv 2(\bmod 3)}}^{\infty} \sigma(n) q^{4 n}-\left(1-24 \sum_{n=1}^{\infty} \sigma(n) q^{6 n}\right) \\
& +2\left(1-24 \sum_{n=1}^{\infty} \sigma(n) q^{12 n}\right)+8 \sum_{n=1}^{\infty} \sigma(n) q^{3 n}+4 \sum_{n=1}^{\infty} c(n) q^{n} \\
& n \equiv 1(\bmod 2) \\
& =1+24 \sum_{n=1}^{\infty} \sigma(n / 6) q^{n}-48 \sum_{n=1}^{\infty} \sigma(n / 12) q^{n} \\
& +\sum_{\substack{n=1 \\
n \equiv 1(\bmod 3)}}^{\infty}(2 \sigma(n)-8 \sigma(n / 4)) q^{n}+\sum_{\substack{n=1 \\
n \equiv 2(\bmod 3)}}^{\infty}(4 \sigma(n)-16 \sigma(n / 4)) q^{n} \\
& +8 \sum_{\substack{n=1 \\
n \equiv 1(\bmod 2)}}^{\infty} \sigma(n / 3) q^{n}+4 \sum_{n=1}^{\infty} c(n) q^{n} \text {. }
\end{aligned}
$$

Equating the coefficients of $q^{n}(n \in \mathbb{N})$, we obtain

$$
N(1,1,1,9 ; n)= \begin{cases}2 \sigma(n)+4 c(n) & \text { if } n \equiv 1(\bmod 6), \\ 4 \sigma(n)-16 \sigma(n / 4) & \text { if } n \equiv 2(\bmod 6), \\ 8 \sigma(n / 3) & \text { if } n \equiv 3(\bmod 6), \\ 2 \sigma(n)-8 \sigma(n / 4) & \text { if } n \equiv 4(\bmod 6), \\ 4 \sigma(n) & \text { if } n \equiv 5(\bmod 6), \\ 24 \sigma(n / 6)-48 \sigma(n / 12) & \text { if } n \equiv 0(\bmod 6) .\end{cases}
$$

When $n \equiv 0(\bmod 2)$ we have the elementary identity

$$
\sigma(n)-3 \sigma(n / 2)+2 \sigma(n / 4)=0 .
$$

Then

$$
\begin{aligned}
& N(1,1,1,9 ; n)=4 \sigma(n)-16 \sigma(n / 4)=12 \sigma(n)-24 \sigma(n / 2) \\
& \text { if } n \equiv 2(\bmod 6) \text {, } \\
& N(1,1,1,9 ; n)=2 \sigma(n)-8 \sigma(n / 4)=6 \sigma(n)-12 \sigma(n / 2) \\
& \text { if } n \equiv 4(\bmod 6) \text {, } \\
& N(1,1,1,9 ; n)=24 \sigma(n / 6)-48 \sigma(n / 12)=24 \sigma(n / 3)-48 \sigma(n / 6) \\
& \text { if } n \equiv 0(\bmod 6) \text {, }
\end{aligned}
$$

which completes the proof of Theorem 1.4. 
7. Evaluation of $N(1,1,9,9 ; n)$ : Proof of Theorem 1.5. By (1.4), Theorem 2.4, and (2.6)-(2.8), we obtain

$$
\begin{aligned}
\sum_{n=0}^{\infty} N( & 1,1,9,9 ; n) q^{n}=\varphi^{2}(q) \varphi^{2}\left(q^{9}\right) \\
= & \frac{1}{6} \varphi^{4}(q)-\frac{2}{3} \varphi^{4}\left(q^{3}\right)+\frac{3}{2} \varphi^{4}\left(q^{9}\right)+\frac{8}{3} \sum_{n=1}^{\infty} c(n) q^{n} \\
= & \frac{1}{6}\left(1+\sum_{n=1}^{\infty}(8 \sigma(n)-32 \sigma(n / 4)) q^{n}\right) \\
& -\frac{2}{3}\left(1+\sum_{n=1}^{\infty}(8 \sigma(n / 3)-32 \sigma(n / 12)) q^{n}\right) \\
& +\frac{3}{2}\left(1+\sum_{n=1}^{\infty}(8 \sigma(n / 9)-32 \sigma(n / 36)) q^{n}\right)+\frac{8}{3} \sum_{n=1}^{\infty} c(n) q^{n} \\
= & +\sum_{n=1}^{\infty}\left(\frac{4}{3} \sigma(n)-\frac{16}{3} \sigma(n / 3)-\frac{16}{3} \sigma(n / 4)\right. \\
& \left.+12 \sigma(n / 9)+\frac{64}{3} \sigma(n / 12)-48 \sigma(n / 36)+\frac{8}{3} c(n)\right) q^{n} .
\end{aligned}
$$

Equating the coefficients of $q^{n}(n \in \mathbb{N})$, and recalling that $c(n)=0$ if $n \not \equiv 1$ $(\bmod 6)$, we obtain

$$
\begin{aligned}
& N(1,1,9,9 ; n) \\
& = \begin{cases}\frac{4}{3} \sigma(n)+\frac{8}{3} c(n) & \text { if } n \equiv 1(\bmod 6), \\
\frac{4}{3} \sigma(n)-\frac{16}{3} \sigma(n / 4) & \text { if } n \equiv 2,4(\bmod 6), \\
\frac{4}{3} \sigma(n)-\frac{16}{3} \sigma(n / 3)+12 \sigma(n / 9) & \text { if } n \equiv 3(\bmod 6), \\
\frac{4}{3} \sigma(n) & \text { if } n \equiv 5(\bmod 6), \\
\frac{4}{3} \sigma(n)-\frac{16}{3} \sigma(n / 3) & \\
-\frac{16}{3} \sigma(n / 4)+12 \sigma(n / 9) & \\
+\frac{64}{3} \sigma(n / 12)-48 \sigma(n / 36) & \text { if } n \equiv 0(\bmod 6) .\end{cases}
\end{aligned}
$$

When $n \equiv 0(\bmod 3)$ we have the elementary identity

$$
\sigma(n)-4 \sigma(n / 3)+3 \sigma(n / 9)=0 .
$$

Then, by (7.1), (6.2) and (7.2), we obtain

$$
\begin{aligned}
N(1,1,1,9 ; n) & =\frac{4}{3} \sigma(n)-\frac{16}{3} \sigma(n / 4)=4 \sigma(n)-8 \sigma(n / 2) \quad \text { if } n \equiv 2,4(\bmod 6), \\
N(1,1,1,9 ; n) & =\frac{4}{3} \sigma(n)-\frac{16}{3} \sigma(n / 4)+12 \sigma(n / 9) \\
& =8 \sigma(n / 9) \quad \text { if } n \equiv 3(\bmod 6),
\end{aligned}
$$




$$
\begin{aligned}
N(1,1,1,9 ; n)= & \frac{4}{3} \sigma(n)-\frac{16}{3} \sigma(n / 3)-\frac{16}{3} \sigma(n / 4) \\
& +12 \sigma(n / 9)+\frac{64}{3} \sigma(n / 12)-48 \sigma(n / 36) \\
= & 8 \sigma(n / 9)-32 \sigma(n / 36) \quad \text { if } n \equiv 0(\bmod 6),
\end{aligned}
$$

which completes the proof of Theorem 1.5.

8. Evaluation of $N(1,9,9,9 ; n)$ : Proof of Theorem 1.6. By (1.4) and Theorem 2.3, we obtain

$$
\begin{aligned}
& \sum_{n=0}^{\infty} N(1,9,9,9 ; n) q^{n}=\varphi(q) \varphi^{3}\left(q^{9}\right) \\
& \quad=\varphi^{2}(q) \varphi^{2}\left(q^{9}\right)-\frac{1}{3} \varphi^{3}(q) \varphi\left(q^{9}\right)+\frac{1}{3} \varphi^{4}\left(q^{3}\right) \\
& =\sum_{n=0}^{\infty} N(1,1,9,9 ; n) q^{n}-\frac{1}{3} \sum_{n=0}^{\infty} N(1,1,1,9 ; n) q^{n}+\frac{1}{3} \sum_{n=0}^{\infty} N(1,1,1,1 ; n) q^{3 n} .
\end{aligned}
$$

Equating the coefficients of $q^{n}(n \in \mathbb{N})$, we obtain

(8.1) $N(1,9,9,9 ; n)=N(1,1,9,9 ; n)-\frac{1}{3} N(1,1,1,9 ; n)+\frac{1}{3} N(1,1,1,1 ; n / 3)$.

From (2.7) we have

$$
N(1,1,1,1 ; n / 3)=8 \sigma(n / 3)-32 \sigma(n / 12), \quad n \in \mathbb{N} .
$$

By Theorem 1.4, Theorem 1.5 and (8.2), we obtain

$$
\begin{aligned}
& N(1,9,9,9 ; n) \\
& = \begin{cases}\frac{2}{3} \sigma(n)+\frac{4}{3} c(n) & \text { if } n \equiv 1(\bmod 6), \\
0 & \text { if } n \equiv 2,5(\bmod 6), \\
8 \sigma(n / 9) & \text { if } n \equiv 3(\bmod 6), \\
2 \sigma(n)-4 \sigma(n / 2) & \text { if } n \equiv 4(\bmod 6), \\
-\frac{16}{3} \sigma(n / 3)+16 \sigma(n / 6)+8 \sigma(n / 9) & \\
-\frac{32}{3} \sigma(n / 12)-32 \sigma(n / 36) & \text { if } n \equiv 0(\bmod 6) .\end{cases}
\end{aligned}
$$

If $n \equiv 0(\bmod 6)$, then $n \equiv 0(\bmod 2)$, and so by $(6.2)$, we have

$$
-\frac{16}{3} \sigma(n / 3)+16 \sigma(n / 6)-\frac{32}{3} \sigma(n / 12)=0,
$$

which completes the proof of Theorem 1.6.

We conclude the paper by noting that for $n \in \mathbb{N}$,

$$
N(1,1,9,9 ; n)= \begin{cases}N(1,1,1,1 ; n / 9) & \text { if } n \equiv 0(\bmod 3), \\ 2 N(1,9,9,9 ; n) & \text { if } n \equiv 1(\bmod 3), \\ \frac{1}{3} N(1,1,1,9 ; n) & \text { if } n \equiv 2(\bmod 3),\end{cases}
$$

which follows from Theorem 1.4, Theorem 1.6 and (8.2). This result can also be proved independently of Theorems 1.4 and 1.6 in a similar manner to the proof of Lemma 5.1. 
Acknowledgments. I would like to thank Professor Kenneth S. Williams for helpful discussions during the preparation of this paper.

\section{References}

[1] A. Alaca, Ş. Alaca, M. F. Lemire and K. S. Williams, Nineteen quaternary quadratic forms, Acta Arith. 130 (2007), 277-310.

[2] - , 一, - - - Theta function identities and representations by certain quaternary quadratic forms, Int. J. Number Theory 4 (2008), 219-239.

[3] A. Alaca, S.. Alaca and K. S. Williams, On the two-dimensional theta functions of the Borweins, Acta Arith. 124 (2006), 177-195.

[4] - , 一, - Evaluation of the convolution sums $\sum_{l+12 m=n} \sigma(l) \sigma(m)$ and $\sum_{3 l+4 m=n}$ $\sigma(l) \sigma(m)$, Adv. Theoret. Appl. Math. 1 (2006), 27-48.

[5] S. Alaca and K. S. Williams, Evaluation of the convolution sums $\sum_{l+6 m=n} \sigma(l) \sigma(m)$ and $\sum_{2 l+3 m=n} \sigma(l) \sigma(m)$, J. Number Theory 124 (2007), 491-510.

[6] B. C. Berndt, Ramanujan's Notebooks, Part III, Springer, New York, 1991.

[7] C. G. J. Jacobi, Fundamenta Nova Theoriae Functionum Ellipticarum, Regiomonti, 1829. See also: Gesammelte Werke, Vol. I, Chelsea, New York, 1969, 49-239.

[8] F. Klein und R. Fricke, Vorlesungen über die Theorie der elliptischen Modulfunktionen, Vols. I, II, Teubner, Leipzig, 1890, 1892.

[9] G. Lomadze, Über die Darstellung der Zahlen durch einige quaternäre quadratische Formen, Acta Arith. 5 (1959), 125-170.

[10] - The number of representations of numbers by quadratic forms with four variables, Sakharth. SSR Mecn. Akad. Math. Ins. Šrom. 40 (1971), 106-139 (in Russian, Georgian summary).

[11] L. J. Mordell, On Mr Ramanujan's empirical expansions of modular functions, Proc. Cambridge Philos. Soc. 19 (1917), 117-124.

[12] K. Petr, Bemerkung zu einer Gaussschen Formel über die Thetafunktionen, Prag. Ber. 1904, Nr. 37, 6 pages (Jahrbuch database JFM 35.0466.03).

[13] S. Ramanujan, On certain arithmetical functions, Trans. Cambridge Philos. Soc. 22 (1916), 159-184.

[14] —, Collected Papers, AMS Chelsea Publ., Providence, RI, 2000.

School of Mathematics and Statistics

Carleton University

Ottawa, Ontario, Canada K1S 5B6

E-mail: aalaca@math.carleton.ca

Received on 23.5.2008

and in revised form on 5.8.2008 